\title{
Effect of extending the interval from Presynch to initiation of Ovsynch in a Presynch-Ovsynch protocol on fertility of timed artificial insemination services in lactating dairy cows
}

\author{
J. O. Giordano,, ${ }^{* 1}$ M. J. Thomas,† G. Catucuamba,† M. D. Curler,† R. Wijma, ${ }^{*}$ M. L. Stangaferro, ${ }^{*}$ \\ and M. Masello* \\ *Department of Animal Science, Cornell University, Ithaca, NY 14853 \\ †Dairy Health and Management Services LLC, Lowville, NY 13367
}

\section{ABSTRACT}

The specific objective of this study was to determine if increasing the interval between the Presynch and Ovsynch portion of the Presynch-Ovsynch protocol (Presynch: $\mathrm{PGF}_{2 \alpha}-14 \mathrm{~d}-\mathrm{PGF}_{2 \alpha}$ and Ovsynch: GnRH-7 d-PGF ${ }_{2 \alpha}-56$ h-GnRH-16-20 h-timed artificial insemination) from 12 to $14 \mathrm{~d}$ would reduce the fertility of lactating dairy cows not detected in estrus after Presynch that receive timed artificial insemination (TAI). Cows from 4 commercial dairy farms $(\mathrm{n}=3,165)$ were blocked by parity (primiparous vs. multiparous) and randomly assigned to a $12(\mathrm{PSOv} 14-12 ; \mathrm{n}=1,566)$ or $14 \mathrm{~d}$ (PSOv14-14; $\mathrm{n}=1,599)$ interval between the second $\mathrm{PGF}_{2 \alpha}$ (PGF) injection of Presynch (P2) and the beginning of Ovsynch. Cows detected in estrus any time between P2 and the day of the TAI were inseminated (AIED group). From a subgroup of cows (177 and 150 in PSOv14-12 and PSOv14-14, respectively), ovarian parameters and ovulation were evaluated through determination of concentrations of progesterone (P4) in blood and transrectal ultrasonography at the time of the first GnRH (GnRH1) and the PGF injection of Ovsynch. Overall, $52.8 \%(\mathrm{n}=1,671)$ of the cows were AIED, whereas $47.2 \%(\mathrm{n}=1,494)$ received TAI. For cows that received TAI, pregnancies per artificial insemination $39 \mathrm{~d}$ after artificial insemination were similar for PSOv14-12 (36.3\%) and PSOv14-14 (36.0\%) but were greater for primiparous $(41.5 \%)$ than multiparous cows (33.6\%). Pregnancy loss from 39 to $105 \mathrm{~d}$ after artificial insemination was similar for PSOv14-12 (4.8\%) and PSOv14-14 (8.6\%), for primiparous (6.4\%) and multiparous cows (7.0\%), but a tendency for a treatment by parity interaction was observed. Both treatments had a similar proportion of cows with a follicle $\geq 10 \mathrm{~mm}$ and similar follicle size at GnRH1; however, the ovulatory response to $\mathrm{GnRH}$ was greater

Received July 7, 2015.

Accepted September 2, 2015.

${ }^{1}$ Corresponding author: jog25@cornell.edu for PSOv14-12 (62.2\%) than PSOv14-14 (46.4\%). A greater proportion of cows with a functional corpus luteum (75.3 vs. $65.6 \%$ ) and greater concentrations of P4 (3.9 vs. $3.3 \mathrm{ng} / \mathrm{mL})$ at GnRH1 in PSOv14-14 than PSOv14-12 may have compensated for the reduction in fertility expected due to reduced ovulatory response to GnRH1. We concluded that extending the interval from Presynch to Ovsynch from 12 to 14 reduced ovulatory response to GnRH1 but did not reduce the fertility of cows that received TAI when cows were inseminated in estrus after presynchronization. Thus, farms that combine AIED and TAI during the Presynch-Ovsynch protocol may use a 14-d interval between Presynch and Ovsynch to simplify their management without reducing fertility of cows receiving TAI.

Key words: Presynch-Ovsynch, estrus detection, timed artificial insemination, dairy cow

\section{INTRODUCTION}

Presynch-Ovsynch (Presynch: $\mathrm{PGF}_{2 \alpha}-14$ d-PGF $\mathrm{PG}_{2 \alpha}$; Ovsynch: GnRH-7 d-PGF ${ }_{2 \alpha}-56$ h-GnRH-16-20 h-TAI; Moreira et al., 2001) is one of the most widely used protocols to synchronize estrus and ovulation for first AI service postpartum in lactating dairy cows in the United States (Caraviello et al., 2006; Ferguson and Skidmore, 2013). Based on the expected pattern of estrus expression and ovulation after the second $\mathrm{PGF}_{2 \alpha}$ (PGF) injection of Presynch, it has been proposed that the ideal time to initiate Ovsynch to maximize fertility of timed AI (TAI) services is at least 10 and no more than 12 d after Presynch (Moreira et al., 2001; Galvão et al., 2007; Colazo et al., 2013). From a practical perspective, a 10-, 11-, or 12-d interval from Presynch to Ovsynch requires handling cows to administer hormone injections during at least 2 to 4 different days of the week, which increases labor needs, disrupts cow time budgets, and in some cases may reduce protocol compliance. To simplify management, some dairy farms prefer to use the Presynch-Ovsynch protocol with a 14-d interval from the second PGF injection 
of Presynch to the first GnRH injection of Ovsynch (GnRH1). All hormone injections, except for the last GnRH of Ovsynch, are administered on $1 \mathrm{~d}$ of the week. A potential caveat of this simplified strategy is that it may reduce fertility of TAI services. In this regard, Galvão et al. (2007) demonstrated that extending the interval from Presynch to Ovsynch from 11 to $14 \mathrm{~d}$ reduced fertility of TAI services by 7 percentage points. In this study, the Ovsynch portion of the protocol was modified by replacing the second GnRH injection with estradiol cypionate (Heatsynch), and more importantly all cows received a TAI service. Many herds, however, adopt the Presynch-Ovsynch program because it enables the insemination of a high proportion of cows after a detected estrus (AIED) immediately after the end of the voluntary waiting period, usually coincident with the second PGF injection of Presynch. In well-managed dairy herds with effective estrus detection programs, the percentage of cows AIED after Presynch is $\sim 50 \%$, although it could vary between 20 to $70 \%$ (Lima et al., 2009; Chebel et al., 2010; Fricke et al., 2014). Thus, in dairy farms that prefer to inseminate cows after a detected estrus during Presynch-Ovsynch, the Ovsynch portion of the protocol is required to synchronize ovulation in cows not inseminated after Presynch because they fail to express estrus or are not detected in estrus.

Extending the interval from Presynch to Ovsynch to $14 \mathrm{~d}$ would be counterintuitive based on the known reduction in fertility when all cows receive TAI (Galvão et al., 2007). This notion is, however, valid under the assumption that cows not AIED after Presynch ovulate and the timing of ovulation is similar to that of cows that displayed estrus after the second PGF of Presynch [i.e., within 3 to $6 \mathrm{~d}$ based on (Chebel et al., 2006)]. In that case many cows would be approximately on d 8 to 11 of the estrous cycle when the response to the first GnRH injection of Ovsynch is expected to be poor. At this stage of the estrous cycle, the dominant follicle of the first follicular wave would be either atretic or becoming atretic, and thereby not be as responsive to the LH surge caused by GnRH. Failure to ovulate in response to the first GnRH injection of Ovsynch leads to reduced overall synchrony of ovulation and fertility after TAI in dairy cows (Chebel et al., 2006; Galvão and Santos, 2010; Giordano et al., 2012c). Nevertheless, an intriguing possibility is that when cows are AIED after Presynch, the duration of the interval between Presynch and Ovsynch is less relevant because cows that reach Ovsynch would be less likely to be properly presynchronized. A substantial proportion of the cows not detected in estrus may have not ovulated or ovulated outside the expected timeframe after Presynch and would thereby not be at the expected stage of the estrous cycle when they receive the first $\mathrm{GnRH}$ of
Ovsynch. Remarkably, no large-scale field study has been conducted to evaluate the reproductive performance and physiological response of cows synchronized with Presynch-Ovsynch with $14 \mathrm{~d}$ versus shorter intervals from Presynch to Ovsynch when cows are AIED after Presynch. We hypothesize that extending the interval from Presynch to Ovsynch by $2 \mathrm{~d}$ (from 12 to 14 d) will not reduce the fertility of cows receiving a TAI service when cows detected in estrus after Presynch are inseminated.

Thus, the specific objective of this study was to determine if increasing the interval between the Presynch and Ovsynch portion of the Presynch-Ovsynch protocol from 12 to $14 \mathrm{~d}$ would reduce the fertility of lactating dairy cows not detected in estrus after Presynch and receive TAI after completion of the Ovsynch protocol.

\section{MATERIALS AND METHODS}

\section{Farms and Management}

Lactating Holstein cows from 4 commercial dairy farms (A through D) in northern New York were enrolled in this study conducted from April 2013 to June 2014. The approximate number of milking cows during the study period was $470,1,770,570$, and 750 for farms A, B, C, and D, respectively. In farms A and $\mathrm{B}$, cows were housed in free-stall barns with 6 rows of stalls with mattresses covered with sawdust, whereas in farms $\mathrm{C}$ and $\mathrm{D}$ cows were housed in barns with 4 rows of stalls and sand bedding. Cows were fed a TMR to meet or exceed the nutritional requirements of lactating Holstein cows producing 38 to $44 \mathrm{~kg}$ of milk per day (NRC, 2001). Feed was delivered once daily and cows had free access to feed and water. Cows were milked 3 times daily and received injections of recombinant bST (Posilac, 500 mg; Elanco Animal Health, Indianapolis, IN) at 14-d intervals starting at $\sim 60$ to 70 DIM until dry-off. Health management protocols and veterinary services were similar for the 4 farms because all protocols were standardized by Dairy Health and Management Services LLC (Lowville, NY).

\section{Treatments}

Every week, cows $(\mathrm{n}=3,165$; farm $\mathrm{A}=514$, farm $\mathrm{B}=1,946$, farm $\mathrm{C}=467$, and farm $\mathrm{D}=238$ ) that reached $21 \pm 3$ DIM were automatically enrolled in the study by the on-farm software (DairyComp305, Valley Ag, Tulare, CA) through a command that randomly assigned a treatment code to cows within parity groups (primiparous and multiparous). All cows received the Presynch-Ovsynch protocol (Presynch: PGF-14 d-PGF and Ovsynch: GnRH-7 d-PGF-56 h-GnRH-16 to 20 
h-TAI) for synchronization of estrus and ovulation. The experimental treatments differed in the interval between the second PGF injection of Presynch and the beginning of the Ovsynch protocol. For cows enrolled in Presynch-Ovsynch 14-12 (PSOv14-12; $\mathrm{n}=1,566$ ) the interval between the Presynch and Ovsynch portion of the protocol was $12 \mathrm{~d}$, whereas for cows enrolled in Presynch-Ovsynch 14-14 (PSOv14-14; $\mathrm{n}=1,599$ ) the interval was $14 \mathrm{~d}$ (Figure 1). To maintain the same DIM (i.e., $73 \pm 3$ ) and day of the week for TAI (i.e., Thursday or Friday), the Presynch portion of PresynchOvsynch was initiated $2 \mathrm{~d}$ later for cows in PSOv14-12 (i.e., $37 \pm 3$ ) than in PSOv14-14 (i.e., $35 \pm 3$; Figure 1). The synthetic analog of PGF, Cloprostenol, was used at a dose of $500 \mu \mathrm{g}$ per injection $(250 \mu \mathrm{g} / \mathrm{mL}$ estroPlan, Parnell Technologies Pty, Alexandria, Australia), whereas the $\mathrm{GnRH}$ product was gonadorelin used at a dose of $100 \mu \mathrm{g}$ per injection $(100 \mu \mathrm{g} / \mathrm{mL}$, Gonabreed, Parnell Technologies Pty). All hormone injections were given intramuscularly by farm personnel.

Cows detected in estrus any time between the second PGF injection of Presynch and the day of the TAI were inseminated (Figure 1). Any cow inseminated in estrus before the end of the voluntary waiting period, coincident with the second PGF injection of Presynch, was removed from the study. Detection of estrus was performed by visual observation (i.e., standing behavior and secondary signs of estrus such as redness of the vulva, vaginal discharge, and restlessness) in all farms except farm B, which combined visual observation with increased physical activity as determined by pedometers (Afimilk, Kibbutz Afikim, Israel).

\section{Blood Sampling and Progesterone Analysis}

From a subgroup of cows in farms A, B, and D, blood samples for determination of concentrations of progesterone (P4) were collected by puncture of the caudal vein or artery using serum separator evacuated tubes (Vacutainer, BD, Franklin Lakes, NJ) at the time of the first GnRH (GnRH1) and the PGF injection of Ovsynch (Figure 1). After collection, samples were refrigerated at $4^{\circ} \mathrm{C}$ until they were transported to the laboratory for further processing (centrifugation at $2,000 \times g, 15 \mathrm{~min}$, room temperature) and stored at $-20^{\circ} \mathrm{C}$ until assayed. Concentrations of $\mathrm{P} 4$ in serum were estimated using a solid-phase, no extraction RIA (Coat-a-count, Diagnostics Products Corporation, Los Angeles, CA). Two quality control samples with concentrations of $\mathrm{P} 4$ representative of the diestrus (high

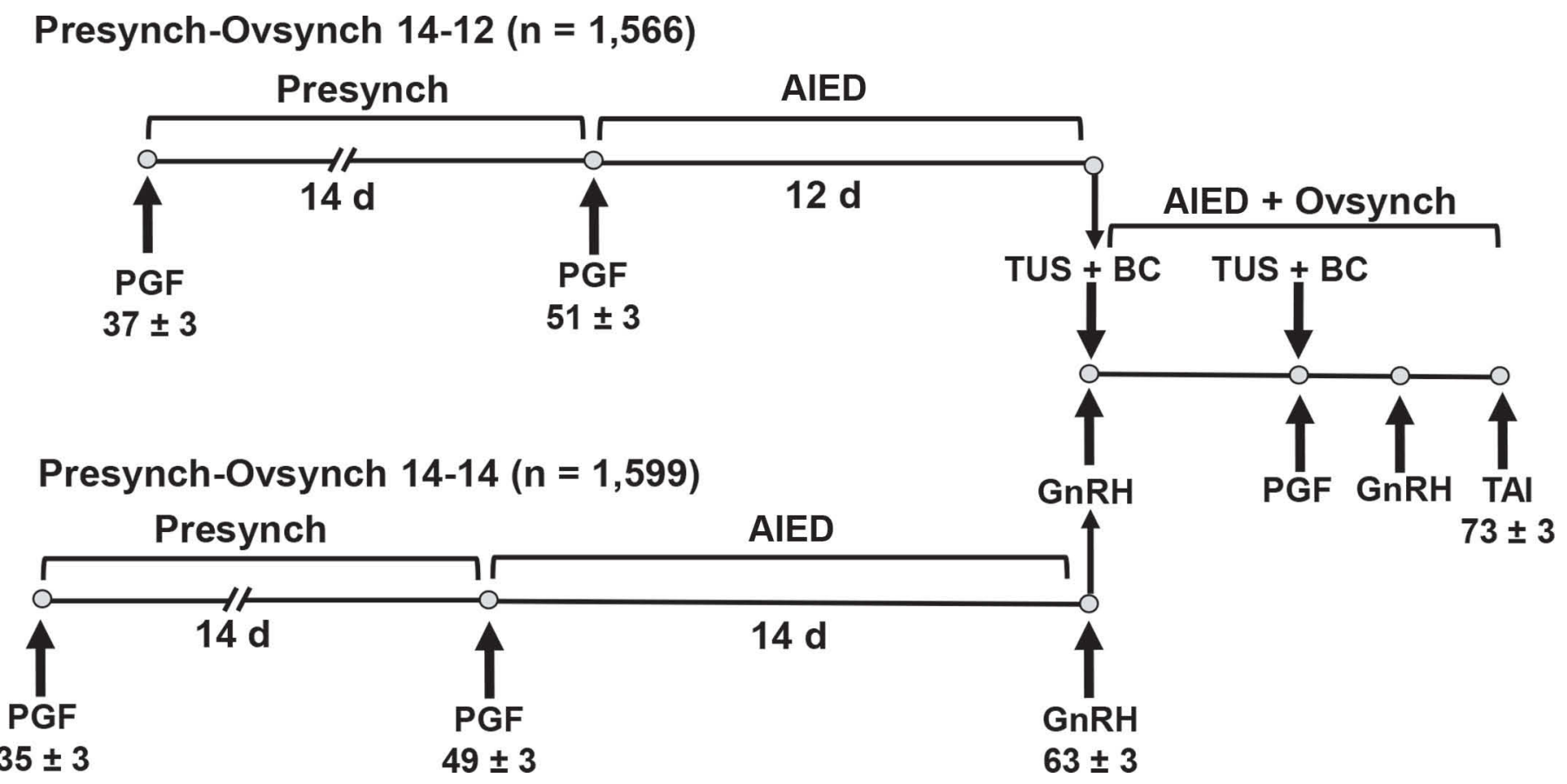

Figure 1. Schematic representation of experimental procedures. Lactating dairy cows were randomly assigned to receive the PresynchOvsynch protocol (Presynch: PGF-14 d-PGF and Ovsynch: GnRH-7 d-PGF-56 h-GnRH- 16 to 20 h-TAI) with an interval of 12 (PSOv14-12; n $=1,566$ ) or $14 \mathrm{~d}$ (PSOv14-14; $\mathrm{n}=1,599)$ from the second PGF injection of Presynch to the first GnRH injection of Ovsynch. To maintain the same DIM (i.e., $73 \pm 3$ ) and day of the week for TAI (i.e., Thursday), cows in PSOv14-12 began the protocol at $37 \pm 3$ DIM, whereas cows in PSOv14-14 began the protocol at $35 \pm 3$ DIM. Cows detected in estrus any time between the second PGF injection of Presynch and the day of the TAI were inseminated. Transrectal ultrasonography of the ovaries was conducted and a blood sample collected at the time of the first $\mathrm{GnRH}$ and the PGF injection of the Ovsynch portion of the Presynch-Ovsynch protocol. TUS = transrectal ultrasound, BC = blood collection. 
concentration) and proestrus (low concentration) phase of the estrous cycle were included twice (beginning and end) across each assay to determine the variation within and across assays. The average sensitivity of the assay was $0.03 \mathrm{ng} / \mathrm{mL}$ of $\mathrm{P} 4$. Intra- and interassay $\mathrm{CV}$ for the high $\mathrm{P} 4$ concentration sample $($ mean $=7.6 \mathrm{ng} / \mathrm{mL}$ ) for the 10 assays run was 7.9 and $10.4 \%$, respectively. Intra- and interassay $\mathrm{CV}$ for the low $\mathrm{P} 4$ concentration sample (mean $=0.4 \mathrm{ng} / \mathrm{mL}$ ) for the 10 assays run were 10.4 and $13.6 \%$, respectively.

\section{Ovulatory Response and Pregnancy Status Determination}

Transrectal ultrasonography (TUS) of the ovaries was performed at the time of blood sample collection (Figure 1) to determine the type and size of ovarian structures present at the time of each evaluation and ovulatory response to the first $\mathrm{GnRH}$ injection of Ovsynch. Ultrasonography was performed using portable ultrasound machines fitted with 7 . Five $\mathrm{MHz}$ linear array transducers (Easi-Scan II or Easi-Scan III, BCF Technology Ltd., Livingston, UK). The presence and size of ovarian structures [corpora lutea (CL), follicles, fluid-filled cavities $\geq 25 \mathrm{~mm}$ with or without luteal tissue] were estimated using on-screen background gridlines comprising squares with $10-\mathrm{mm}$ sides. Ovulation in response to GnRH1 was determined at the time of the PGF injection of Ovsynch and was defined as the appearance of at least one new CL on the same ovary where a follicle $\geq 10 \mathrm{~mm}$ was observed at the time of the GnRH injection. All TUS examinations were performed by 1 of 4 bovine veterinary practitioners with 1 to 20 yr of experience performing the technique. The same practitioners performed pregnancy diagnosis at $39 \pm$ $3 \mathrm{~d}$ after AI with TUS. A positive pregnancy outcome was defined after confirmation of the presence of an embryo with a heartbeat and amount of pregnancy fluid in accordance with the stage of pregnancy. Pregnancy reconfirmation was performed at $105 \pm 3 \mathrm{~d}$ after AI with TUS by the same practitioners.

\section{Statistical Analysis}

The experimental design was a complete randomized design with parity (primiparous vs. multiparous) as the stratification factor. The effect of treatment (PSOv1412 vs. PSOv14-14), parity, and other confounders on binomial outcomes [proportion of cows receiving AIED or TAI, pregnancies per AI (P/AI), and pregnancy loss] was analyzed by logistic regression using the GLIMMIX procedure of SAS (version 9.4, SAS Institute Inc., Cary, NC). When appropriate, the least significant difference mean separation test was used to determine differences between least squares means. Explanatory variables offered to all statistical models were treatment, parity, and their respective interaction, whereas models for $\mathrm{P} / \mathrm{AI}$ and pregnancy loss also included season of insemination (cool versus warm) with interactions of appropriate biological interest. Final statistical models were selected by backward elimination of explanatory variables with $P>0.10$ and determination of the lowest value for the Akaike information criterion. Treatment and parity were forced in all models. Farm (A through D) was included as a random effect in all models.

Because farm was included as a random effect in statistical models to evaluate differences in the proportion of cows AIED and fertility, it was not possible to compare differences by farm or to evaluate any potential treatment by farm interaction. Therefore, we conducted a separate analysis to evaluate the proportion of cows AIED and P/AI at $39 \mathrm{~d}$ after AI with models that included treatment, farm, and their interaction as explanatory variables.

Binomial physiological outcomes of interests (proportion of cows with a CL based on TUS and concentrations of P4 at GnRH1 and PGF, proportion of cows with a follicle $\geq 10 \mathrm{~mm}$ at GnRH1 and PGF, ovulatory response to GnRH1, and spontaneous luteolysis during Ovsynch) were evaluated by logistic regression using PROC GLIMMIX of SAS including treatment and parity as explanatory variables and farm as a random effect. Quantitative physiological outcomes of interest (concentrations of P4 at GnRH1 and PGF, follicle size) were analyzed by ANOVA using PROC MIXED of SAS with models that contained treatment and parity as explanatory variables and farm as random effect.

All explanatory variables and their interactions were considered significant if $P \leq 0.05$, whereas $0.05<P$ $\leq 0.10$ was considered a tendency. For simplification of interpretation, means for proportions are reported as arithmetic means obtained with PROC FREQ of $\mathrm{SAS}$, whereas for quantitative variables, means $\pm \mathrm{SE}$ obtained with PROC MEANS of SAS are reported.

\section{RESULTS AND DISCUSSION}

\section{Insemination Pattern and Fertility of Cows After a Detected Estrus}

Overall, the proportion of cows that received insemination after a detected estrus (AIED) from the time of the second PGF injection of Presynch until the day before TAI was $52.8 \%(1,671 / 3,165)$, whereas $47.2 \%$ $(1,494 / 3,165)$ of the cows received a TAI service. Overall, a greater proportion of cows received AIED in the PSOv14-12 compared with the PSOv14-14 treatment (54.8 vs. $50.8 \%$, respectively; $P<0.01$ ), in primiparous 
compared with multiparous cows (61.5 vs. $47.0 \%$, respectively; $P<0.0001$ ), and during the cool compared with during the warm season of the year (54.1 vs. $48.8 \%$, respectively; $P<0.01)$. A tendency $(P=0.09)$ for an interaction between treatment and season of insemination was also detected such that fewer cows were AIED in the PSOv14-14 treatment but only during the warm season of the year. The proportion of cows and distribution of inseminations based on a detected estrus during the protocol followed the expected pattern for cows synchronized with the Presynch-Ovsynch protocol (Figure 2) and is in agreement with previous reports (Chebel et al., 2006; Lima et al., 2009; Chebel et al., 2010). Nevertheless, more cows $(P=0.02)$ received AIED from the time of the second PGF injection of Presynch until GnRH1 in the PSOv14-12 (45.9\%) than in the PSOv14-14 (41.5\%) treatment, which explains the overall differences between treatments for the total proportion of cows AIED. Conversely, from the time of GnRH1 to the PGF injection, and from the time of PGF injection of Ovsynch to the day before TAI, a similar $(P>0.05)$ proportion of cows received AIED.
A plausible explanation for the reduced percentage of cows detected in estrus in PSOv14-14 is the earlier DIM at enrollment into the synchronization protocol. Although a 2-d difference between treatments is rather small, it is likely that more cows in PSOv14-14 did not present a CL or presented a CL less responsive to the first PGF injection of Presynch because of a recent ovulation. Lack of a CL or failure to induce complete luteal regression of a less mature CL may have altered the pattern of estrus expression and ovulation after the first PGF injection. As a result, cows may have had a reduced response to the second PGF injection of Presynch, thereby reducing the likelihood of estrus expression.

The proportion of cows receiving AIED is in the range reported for lactating Holstein cows in free-stall barns (Lima et al., 2009; Chebel et al., 2010; Chebel and Santos, 2010; Fricke et al., 2014), but it was affected $(P<0.001)$ by farm as observed in previous studies (Chebel et al., 2010). Farm B (58.8\%) had the greatest proportion of cows AIED, followed by farms C (45.6\%) and A (44.0\%), whereas farm D (36.6\%) had the lowest

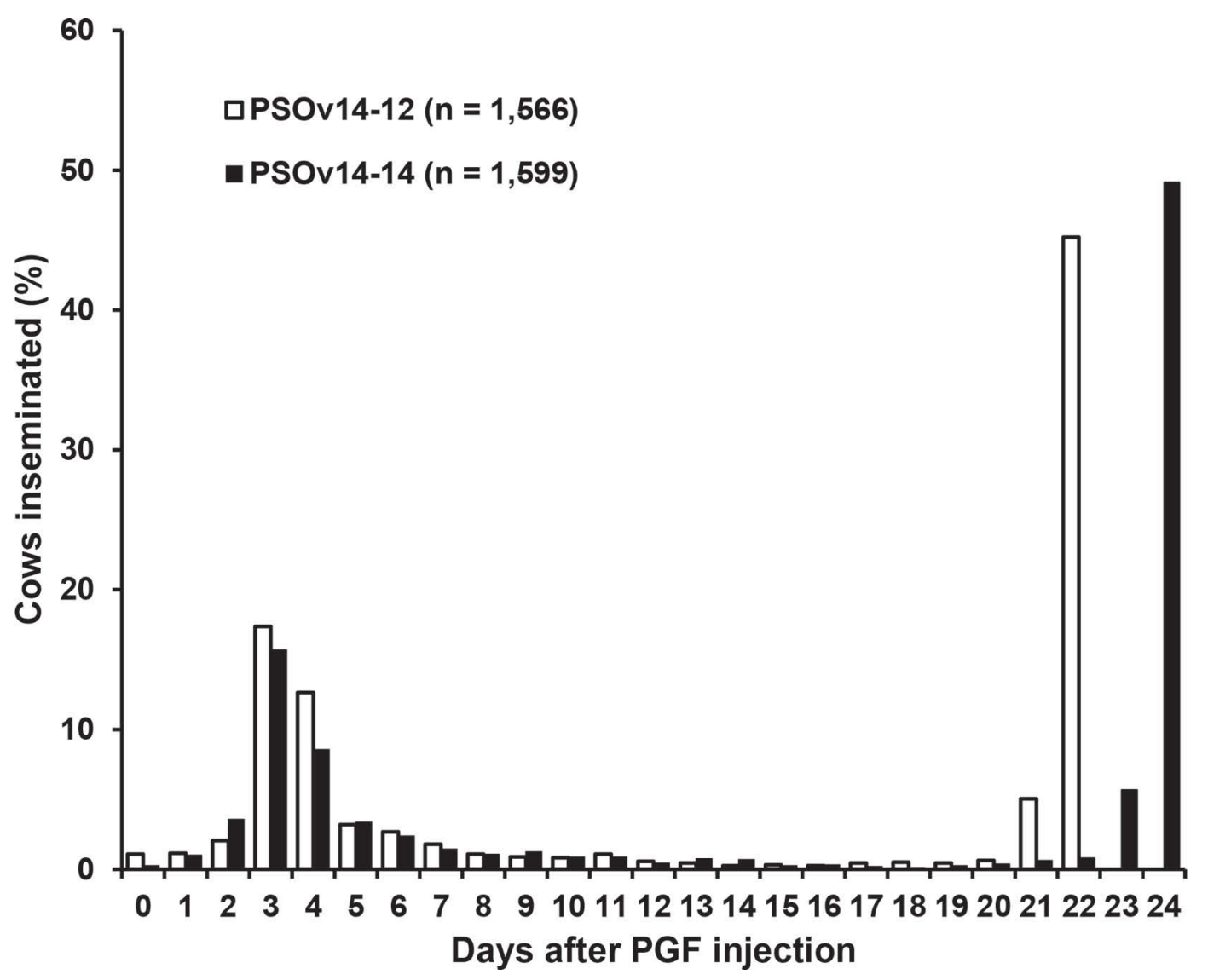

Figure 2. Distribution of AI services from the time of the second prostaglandin $F_{2 \alpha}$ (PGF) injection of Presynch until the day of timed AI in lactating dairy cows enrolled in the Presynch-Ovsynch14-12 (PSOv14-12; n = 1,566) and Presynch-Ovsynch14-14 (PSOv14-14; $\mathrm{n}=1,599)$ protocol. Cows inseminated on d 22 and 24 in PSOv14-12 and PSOv14-14, respectively, received timed AI. 
proportion of cows AIED. Intrinsic differences among herds (e.g., proportion of anovular cows and lameness incidence) as well as differences in methodology (e.g., visual observation only versus visual observation plus physical activity), intensity, and accuracy of estrus detection are the most likely reasons for the observed differences in the proportion of cows detected in estrus among farms.

At 39 d after AI, a treatment by parity interaction $(P<0.01)$ was observed for $\mathrm{P} / \mathrm{AI}$ for cows receiving AIED. Pregnancies per AI were greatest for multiparous cows in PSOv14-14 and primiparous cows in PSOv14-12, intermediate for primiparous cows in PSOv14-14, and lowest for multiparous cows in the PSOv14-12 treatment (Table 1). At $105 \mathrm{~d}$ after AI, P/ AI were also affected by a treatment by parity interaction $(P<0.01)$ with similar trends for $\mathrm{P} / \mathrm{AI}$ differences among treatments. In this case, however, all groups had greater P/AI than multiparous cows in PSOv14-12 (Table 1). The reason for the substantial difference in $\mathrm{P} / \mathrm{AI}$ (9 percentage points) between multiparous cows that received AIED is unclear at the moment. A plausible explanation is that because more cows received AIED in PSOv14-12 (3.5\% more cows), more cows in this treatment were inseminated based on secondary signs of estrus or at the wrong time in relationship to estrus. Nevertheless, this is rather unlikely because a relatively small difference was found in the proportion of cows that received AIED between treatments, AI technicians were blind to treatments, inseminations based on a detected estrus were performed at the same time, and primiparous cows in PSOv14-12 had similar $\mathrm{P} / \mathrm{AI}$ compared with primiparous cows in PSOv14-14. Pregnancy loss from 39 to $105 \mathrm{~d}$ after AI for cows that received AIED tended $(P=0.08)$ to be greater for cows in PSOv14-12 than PSOv14-14, but were not affected by parity $(P=0.74)$ or the treatment by parity interaction $(P=0.29$; Table 1$)$.

\section{Fertility of Cows After TAI}

For cows that received TAI, P/AI 39 d after AI were similar $(P=0.56)$ for PSOv14-12 (36.3\%; 257/708) and PSOv14-14 (36.0\%; 283/786), were affected $(P<$ 0.01 ) by parity ( 41.5 vs. $33.6 \%$ for primiparous and multiparous cows, respectively), but no treatment by parity interaction $(P=0.13$; Table 1$)$ was found. Differences in $\mathrm{P} / \mathrm{AI}$ between treatment and parity groups

Table 1. Fertility of cows receiving their first AI service postpartum after a detected estrus or a timed AI (TAI) after synchronization with the Presynch-Ovsynch protocol with a 12 or $14 \mathrm{~d}$ interval from the second PGF of Presynch to the first GnRH of Ovsynch

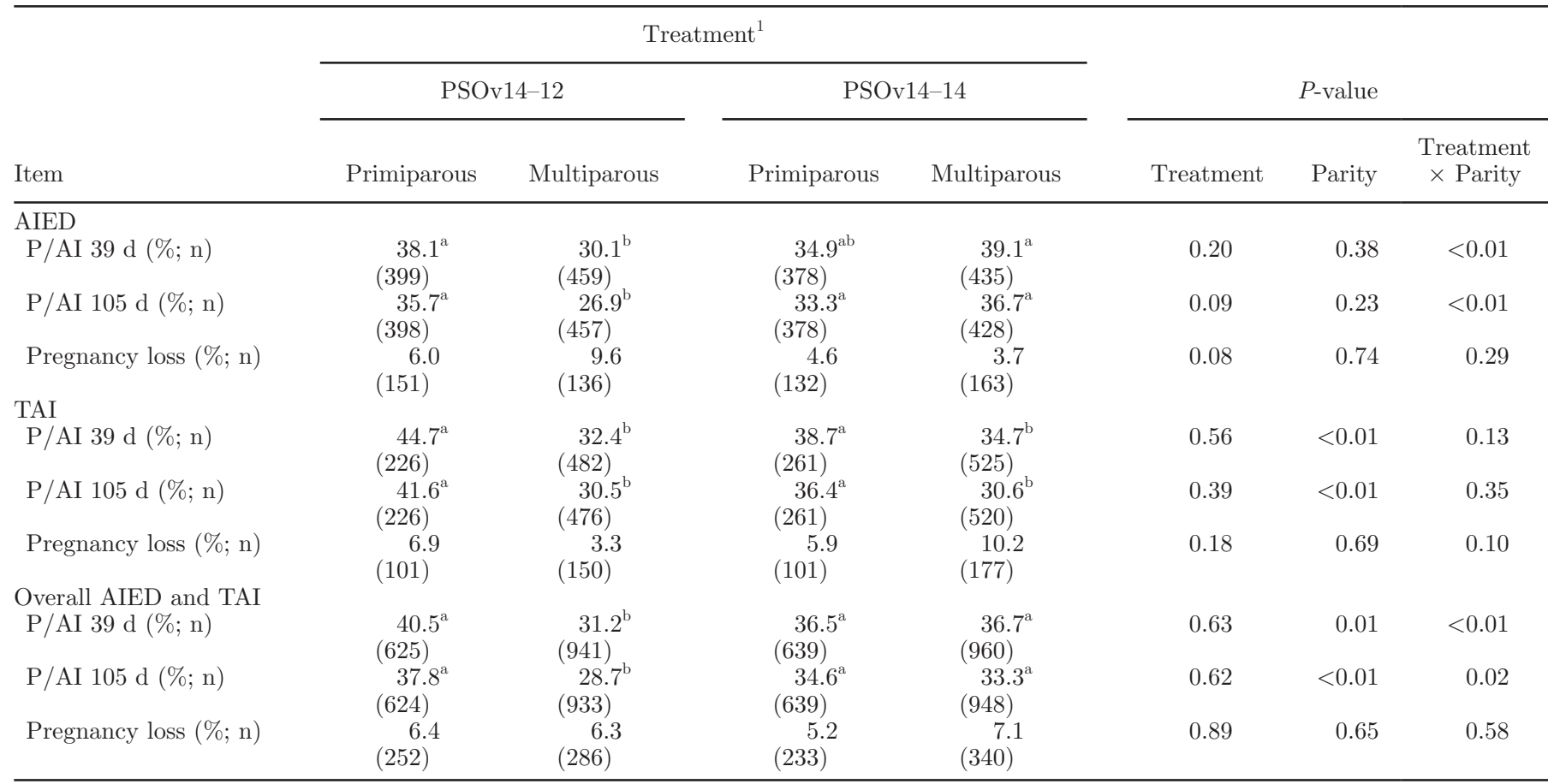

\footnotetext{
${ }^{\mathrm{a}, \mathrm{b}}$ Superscript letters indicate differences within a row based on the least significant difference mean separation test.

${ }^{1}$ Lactating dairy cows received the Presynch-Ovsynch protocol (Presynch: PGF-14 d-PGF and Ovsynch: GnRH-7 d-PGF-56 h-GnRH-16 to 20 h-TAI) with an interval of 12 (PSOv14-12) or $14 \mathrm{~d}$ (PSOv14-14) from the second PGF injection of Presynch to the first GnRH injection of Ovsynch. Cows detected in estrus any time between the second PGF injection of Presynch and the day of the TAI were inseminated. AIED $=$ cows inseminated after a detected estrus; $\mathrm{P} / \mathrm{AI}=$ pregnancies per $\mathrm{AI}$.
} 
were similar at $105 \mathrm{~d}$ after AI (Table 1). Pregnancy loss was similar $(P=0.18)$ for PSOv14-12 $(4.8 \% ; 12 / 251)$ and PSOv14-14 (8.6\%; 24/278), and for primiparous $(6.4 \% ; 13 / 202)$ and multiparous cows $(7.0 \% ; 23 / 327)$, but a tendency for a treatment by parity interaction $(P$ $=0.10$; Table 1) was found.

The lack of difference in $\mathrm{P} / \mathrm{AI}$ for cows that received TAI after synchronization of ovulation with the PSOv14-12 and PSOv14-14 protocol indicate that dairy farms should not expect a reduction in P/AI when the interval from Presynch to Ovsynch is extended by $2 \mathrm{~d}$ from 12 to 14 if cows detected in estrus after Presynch are inseminated. Thus, for dairy farms that use the Presynch-Ovsynch protocol and inseminate cows that express estrus, a 14-d interval between Presynch and Ovsynch may be beneficial to reduce the number of days of the week to administer hormone injections. Our results are in contrast to the reported benefit of reducing the interval from Presynch to Ovsynch from 14 to 11 d (Galvão et al., 2007) but in agreement with a recent study that reported no statistical difference in fertility after $100 \%$ TAI with a Presynch-Cosynch protocol with an interval between Presynch and Cosynch of 10, 12, or $14 \mathrm{~d}$ (Stevenson, 2011). Fertility results after TAI of Stevenson (2011), however, should be interpreted with caution because the study was not designed to compare $\mathrm{P} / \mathrm{AI}$ and thereby had substantially fewer cows per treatment ( 150) than in Galvão et al. (2007).

In spite of a 1-d difference between the short interval treatments (12 vs. $11 \mathrm{~d}$ ) and the use of estradiol cypionate to induce ovulation in Galvão et al. (2007), we hypothesize that the major reason for the discrepancy between the 2 studies is the use of $100 \%$ TAI in Galvão et al. (2007) and a combination of AIED and TAI in our study. The contrast between these 2 studies seems to support the notion that cows inseminated in estrus after Presynch are those that properly respond to presynchronization and as a result would be better synchronized after the Ovsynch portion of the protocol and thereby have better fertility after TAI. In agreement, Fricke et al. (2014) reported that cows which expressed estrus after Presynch but were not inseminated until completion of Presynch-Ovsynch had a 7 percentage point greater $\mathrm{P} / \mathrm{AI}$ (not compared statistically) than cows that were not detected in estrus. Collectively, our data and these previous reports seem to suggest that when all cows proceed to receive TAI regardless of estrus expression after Presynch, an interval of $<14$ $\mathrm{d}$ between Presynch and Ovsynch is beneficial because more cows will be at the appropriate stage of the estrous cycle at the beginning of the Ovsynch protocol. On the other hand, when cows detected in estrus are inseminated after Presynch, those that reach Ovsynch and receive TAI are more likely to be anovular (Chebel and Santos, 2010), not properly presynchronized, or estrus expression impaired due to health or locomotion limitations. Thus, when a substantial proportion of cows are inseminated in estrus after Presynch, 2 extra days from Presynch to Ovsynch are less relevant. In this case, more cows within the subgroup that receive TAI are likely to be at random stages of the estrous cycle when they receive the first GnRH injection of Ovsynch and therefore are less likely to properly respond to the protocol.

The overall $\mathrm{P} / \mathrm{AI}$ for all AI services combined (AIED and TAI $)$ at $39(P<0.01)$ and $105 \mathrm{~d}$ after $\mathrm{AI}(P=$ 0.02 ) were affected by a treatment by parity interaction. At both time points evaluated, $\mathrm{P} / \mathrm{AI}$ were lower for multiparous cows in PSOv14-12 than for the rest of the groups (Table 1). Conversely, pregnancy loss was similar for all treatment and parity groups $(P>0.05$; Table 1). Because most of the reduction in overall $\mathrm{P} /$ AI for multiparous cows in PSOv14-12 in our study can be attributed to the reduction in $\mathrm{P} / \mathrm{AI}$ for cows receiving AIED, herds that obtain similar fertility for cows detected in estrus regardless of the Presynch to Ovsynch interval should not expect any difference in the overall proportion of pregnant cows after first AI service with Presynch-Ovsynch.

When included as a fixed effect in models to evaluate $\mathrm{P} / \mathrm{AI}$ of cows AIED, treatment tended $(P=0.10)$ to affect $\mathrm{P} / \mathrm{AI}$, but neither farm nor the treatment by farm interaction affected P/AI 39 d after AI (Table 2 ). Conversely, for cows that received TAI, farm affected $\mathrm{P} / \mathrm{AI}$ but a treatment by farm interaction was not found (Table 2). The lack of treatment by farm interaction and the very similar P/AI for both treatments across farms indicates that the response of cows to the treatments was consistent across farms in spite of the observed differences in the proportion of cows AIED and TAI. The overall effect of farm on P/AI of cows that received TAI may reflect, as for the proportion of cows that received AIED, intrinsic differences in management, cows, and insemination technique among farms.

\section{Ovarian Parameters and Ovulatory Response}

Data on ovarian parameters and ovulatory response to GnRH1 are depicted in Table 3.

Overall, a high proportion of cows $(\sim 70 \%)$ had a CL detected by TUS or based on blood P4 concentration. These observations could be explained by the failure to detect cows in estrus after Presynch, lack of estrus expression in cows that are cycling, or both. The former notion is supported by the inverse relationship between cows with a functional CL at GnRH1 and estrus detection intensity at each farm in our study $(79,75$, and 
Table 2. Pregnancy per AI for cows receiving insemination after a detected estrus (AIED) or timed AI (TAI) for each of the farms enrolled in the study

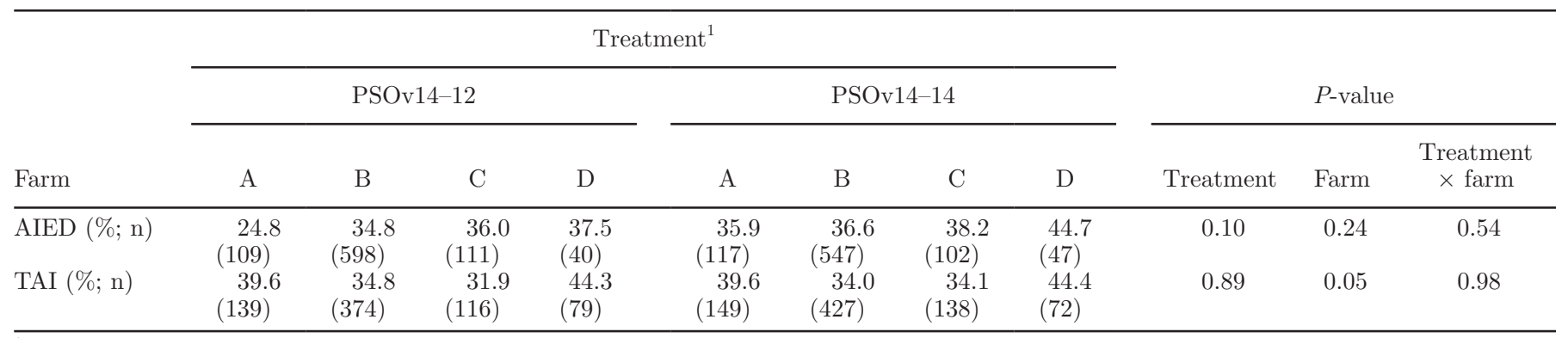

${ }^{1}$ Lactating dairy cows from 4 commercial dairy farms (A through D) received the Presynch-Ovsynch protocol (Presynch: PGF-14 d-PGF and Ovsynch: GnRH-7 d-PGF-56 h-GnRH-16 to 20 h-TAI) with an interval of 12 (PSOv14-12) or 14 d (PSOv14-14) from the second PGF injection of Presynch to the first GnRH injection of Ovsynch. Cows detected in estrus any time between the second PGF injection of Presynch and the day of the timed AI were inseminated. AIED = cows inseminated after a detected estrus.

$62 \%$ of cows with a functional CL at GnRH1, which correspond to $36.6,45.6$, and $58.8 \%$ of the cows detected in estrus during Presynch-Ovsynch in farms D, C, and $\mathrm{B}$, respectively) and the latter by recent studies reporting that $\sim 50$ to $60 \%$ of cows not detected in estrus after Presynch presented a CL at the GnRH1 of Ovsynch (Bruno et al., 2013; Fricke et al., 2014). The proportion of cows with a CL visualized with TUS was similar $(P$ $=0.46)$ between treatments, whereas more $(P=0.05)$ cows had a functional CL based on P4 concentrations $(\geq 1 \mathrm{ng} / \mathrm{mL}$ ) in PSOv14-14 than PSOv14-12. Likely, the reason for the discrepancy between the 2 techniques is that TUS is only useful to determine the presence or absence of luteal tissue, but it is not possible to predict the functionality of the tissue. Indeed, the inaccuracy of a single TUS observation to determine the presence of a functional CL at GnRH1 of Presynch-Ovsynch has been previously reported by others (Silva et al., 2007; Stevenson et al., 2008; Bruno et al., 2013). In our study, overestimation of the presence of a functional CL based on TUS can be explained by the visualization of a newly formed CL that was not producing sufficient P4 to result in circulating $\mathrm{P} 4>1 \mathrm{ng} / \mathrm{mL}$ or the visualization of a regressing or recently regressed CL. We speculate that the former was the most prevalent scenario because 2 additional days of CL growth for cows in

Table 3. Ovarian parameters at the time of the first GnRH injection of the Ovsynch portion of the PresynchOvsynch protocol

\begin{tabular}{|c|c|c|c|}
\hline \multirow[b]{2}{*}{ Item } & \multicolumn{2}{|c|}{ Treatment $^{1}$} & \multirow[b]{2}{*}{$P$-value } \\
\hline & PSOv14-12 & PSOv14-14 & \\
\hline Cows with CL at GnRH1 based on TUS $(\% ; n)$ & $\begin{array}{l}70.1 \\
(177)\end{array}$ & $\begin{array}{l}73.3 \\
(150)\end{array}$ & 0.46 \\
\hline Cows with $\mathrm{P} 4 \geq 1 \mathrm{ng} / \mathrm{mL}$ at $\mathrm{GnRH} 1(\% ; \mathrm{n})$ & $\begin{array}{c}65.6 \\
(180)\end{array}$ & $\begin{array}{l}75.3 \\
(150)\end{array}$ & 0.05 \\
\hline Progesterone concentration $(\mathrm{ng} / \mathrm{mL})$ at $\mathrm{GnRH} 1,(\mathrm{n})$ & $\begin{array}{c}3.3 \\
(180)\end{array}$ & $\begin{array}{c}3.9 \\
(150)\end{array}$ & 0.07 \\
\hline Cows with follicle $\geq 10 \mathrm{~mm}$ at GnRH1 $(\% ; \mathrm{n})$ & $\begin{array}{c}94.7 \\
(171)\end{array}$ & $\begin{array}{c}93.2 \\
(147)\end{array}$ & 0.51 \\
\hline Size of largest follicle $(\mathrm{mm})$ at $\mathrm{GnRH}^{2}(\mathrm{n})$ & $\begin{array}{c}15.7 \\
(171)\end{array}$ & $\begin{array}{c}16.5 \\
(147)\end{array}$ & 0.19 \\
\hline Cows ovulating after $\operatorname{GnRH} 1(\% ; \mathrm{n})$ & $\begin{array}{l}62.2 \\
(172)\end{array}$ & $\begin{array}{l}46.4 \\
(151)\end{array}$ & $<0.01$ \\
\hline \multicolumn{4}{|c|}{$\begin{array}{l}{ }^{1} \text { Lactating dairy cows received the Presynch-Ovsynch protocol (Presynch: PGF-14 d-PGF and Ovsynch: } \\
\text { GnRH-7 d-PGF-56 h-GnRH- } 16 \text { to } 20 \text { h-TAI) with an interval of } 12 \text { (PSOv14-12) or } 14 \text { d (PSOv14-14) from } \\
\text { the second PGF injection of Presynch to the first GnRH injection of Ovsynch. Cows detected in estrus any } \\
\text { time between the second PGF injection of Presynch and the day of the TAI were inseminated. Transrectal } \\
\text { ultrasonography of the ovaries was conducted and a blood sample collected at the time of the first GnRH } \\
\text { and the PGF injection of the Ovsynch portion of the Presynch-Ovsynch protocol. CL = corpus luteum; TUS } \\
=\text { transrectal ultrasonography; P4 = progesterone; GnRH1 = first GnRH injection of the Ovsynch protocol. }\end{array}$} \\
\hline
\end{tabular}


PSOv14-14 allowed more cows to reach concentrations of P4 above $1 \mathrm{ng} / \mathrm{mL}$. Assuming that at least some of the cows not detected in estrus after Presynch ovulated following a similar pattern than those detected in estrus in our study, $\sim 10 \%$ (6\% in PSOv14-12 and $4 \%$ in PSOv14-14) of the cows may have presented a newly formed CL producing $<1 \mathrm{ng} / \mathrm{mL}$ of P4 at GnRH1 because they ovulated 1 to $4 \mathrm{~d}$ before the time of sample collection. Up to $4 \mathrm{~d}$ may be required for $\mathrm{P} 4$ to rise above $1 \mathrm{ng} / \mathrm{mL}$ in some high-producing lactating dairy cows (Sartori et al., 2004; Giordano et al., 2012b). Two additional days of CL growth for cows in PSOv14-14 could also explain, at least in part, the greater concentrations of $\mathrm{P} 4$ for cows in this treatment. Nevertheless, no differences $(P=0.64)$ were found between treatments when only cows with a functional CL at GnRH1 were compared (4.9 vs. $5.1 \mathrm{ng} / \mathrm{mL}$ for PSOv14-12 and PSOv14-14, respectively).

The greater proportion of cows with a CL $(\sim 10 \%)$ based on $\mathrm{P} 4$ and the greater concentrations of $\mathrm{P} 4$ in PSOv14-14 may help explain the lack of difference in fertility for cows receiving TAI in spite of the 15.8 percentage point reduction in cows ovulating after GnRH1 in PSOv14-14 (Table 3). Indeed, the overall effect of ovulation on $\mathrm{P} / \mathrm{AI}$ was smaller than that of the presence of a functional CL at GnRH1 (4.7 vs. 11.2 percentage points, respectively). Whereas cows that ovulated in response to GnRH1 (regardless of treatment) tended to have greater $(P=0.09) \mathrm{P} / \mathrm{AI}$ than cows failing to ovulate (36.4 vs. $31.7 \%$, respectively), the presence of a functional CL at GnRH1 led to greater $(P=0.03)$ differences in $\mathrm{P} / \mathrm{AI}$ (36.8 vs. $25.6 \%$, respectively; Figure 3). Ovulation benefited cows without a CL but did not further improve P/AI of cows with a CL at GnRH1, which were the most prevalent in our study. Interestingly, Galvão et al. (2007) observed similar trends as $\mathrm{P} / \mathrm{AI}$ were 4.5 and 12.2 percentage points numerically greater for cows that ovulated after GnRH1 within the subgroup with or without a CL, respectively. Similar responses have been previously reported for cows with or without a functional CL (Lima et al., 2009) and for cows ovulating or not after GnRH1 of Ovsynch (Giordano et al., 2012c; Colazo et al., 2013). Collectively, these data suggest that the high overall proportion of cows with a functional CL and the greater proportion of cows with a CL in the PSOv14-14 treatment compensated for the reduced ovulatory response to GnRH1. Although both ovulation and the presence of a functional CL at GnRH1 are known to increase P/AI, our observations, and those of other studies (Galvão et al., 2007; Giordano et al., 2012c; Colazo et al., 2013) support the notion that the presence of a functional CL at GnRH1 of Presynch-Ovsynch has a greater effect on $\mathrm{P} / \mathrm{AI}$ than ovulation in response to GnRH1.
The reduction in ovulatory response observed in our study is almost identical to that reported by Galvão et al. (2007). Interestingly, whereas we did not observe a difference in the proportion of cows with a follicle $>10$ $\mathrm{mm}(P=0.51)$ and follicle size $(P=0.19)$ between treatments (Table 3 ), a treatment by parity interaction $(P=0.04)$ for follicle size was observed. Multiparous cows in the PSOv14-14 treatment had greater follicle size $(17.1 \mathrm{~mm})$ than multiparous cows in PSOv14-12 (15.4 mm), whereas primiparous cows in PSOv14-12 $(16.1 \mathrm{~mm})$ and PSOv14-14 $(15.7 \mathrm{~mm})$ had intermediate follicle size and were not different from multiparous cows in either treatment. These differences in follicle size may reflect differences in stage of follicle development at GnRH1 for multiparous cows in PSOv14-14 and PSOv14-12; however, the lack of treatment by parity interaction for ovulatory response to GnRH1 ( $P$ $=0.13)$ supports the notion that follicle size was not a major determinant of ovulatory response to GnRH1. Indeed, the ovulatory response to GnRH1 was not affected $(P=0.61)$ by follicle size at GnRH1 $(57.4,51.3$, 57.8 , and $55.2 \%$ for cows with a follicle $\leq 13,14$ to 16 , 17 to 20 , and $\geq 21 \mathrm{~mm}$ ) for all cows or cows within treatments $(P=0.20)$. Unlike Galvão et al. (2007), who observed the greatest ovulatory response increase in cows with a CL at GnRH1, we observed a similar (13 and 11 percentage point for cows with or without a CL, respectively) reduction in ovulatory response to GnRH1 for cows in PSOv14-14 than PS14-12 (Figure 4). These observations suggest that the stage of follicle development at GnRH1 likely contributed to most of the reduction in ovulatory response to GnRH1 in PSOv14-14 and that follicle size is not an accurate predictor of ovulatory response to the GnRH1 of Presynch-Ovsynch. The presence of a functional CL at GnRH1 caused a 30 percentage point reduction in cows ovulating after GnRH1 across treatments $(P<0.001$; Figure 4), which could explain the remainder of the reduction in ovulation for cows in PSOv14-14 (more cows with a functional CL). Collectively, these observations suggest that the reduced ovulatory response to GnRH1 was the result of the presence of an already atretic follicle unresponsive to an LH surge, an LH surge of lower magnitude due to the suppressive effect of $\mathrm{P} 4$ on $\mathrm{LH}$ release in cows with a CL (Giordano et al., 2012a), or a combination of both.

At the time of the PGF injection of Ovsynch, all ovarian parameters evaluated were similar for both treatments (Table 4). The proportion of cows with a CL determined by TUS and concentrations of P4 was similar for both treatments. A similar trend for overestimation of the presence of a CL based on TUS was observed. Potential reasons for the discrepancy are recent ovulations in cows without a CL at GnRH1, leading 


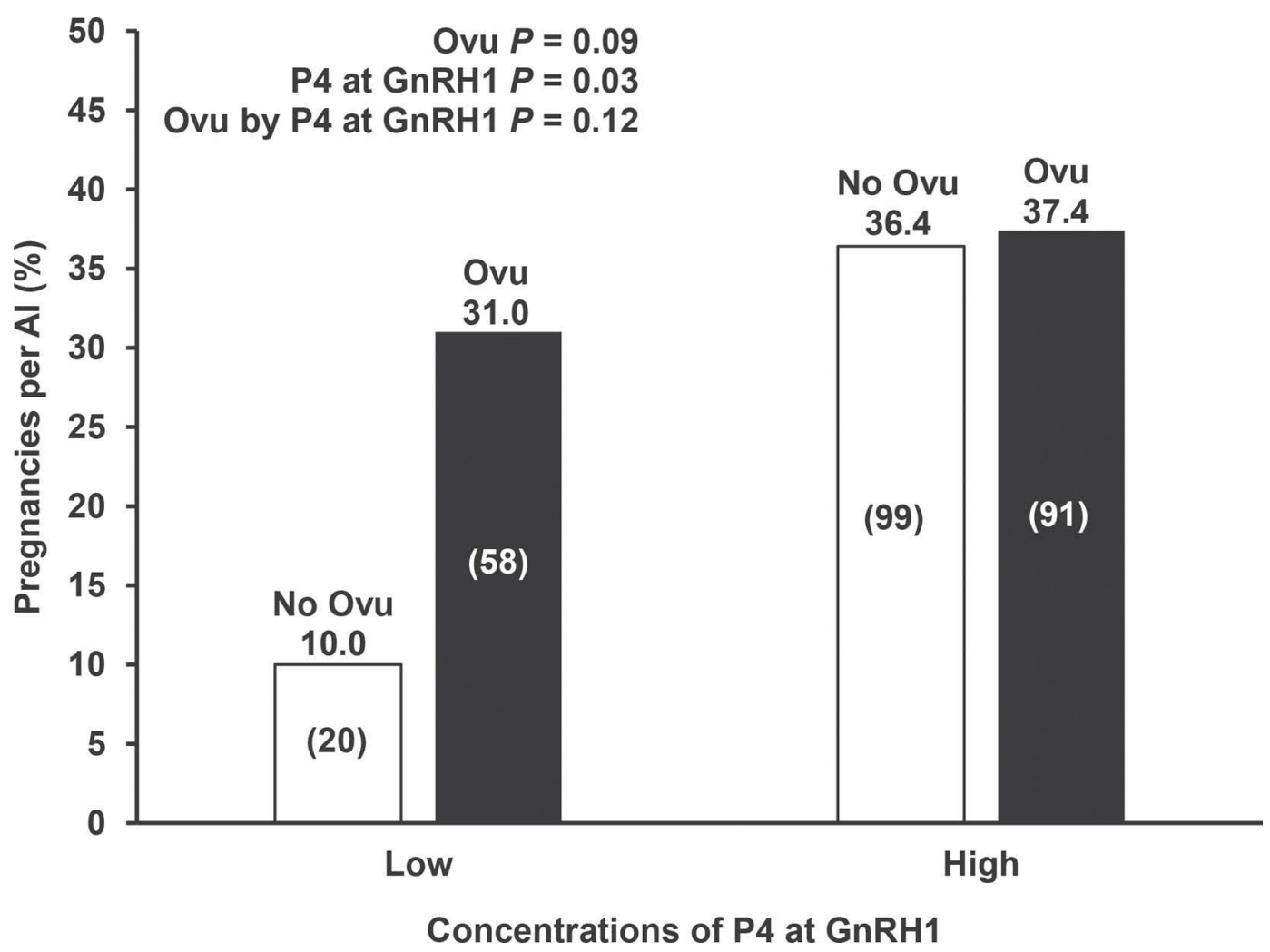

Figure 3. Pregnancies per AI (P/AI) for subgroups of lactating dairy cows created based on the presence of a functional corpus luteum (progesterone, $\mathrm{P} 4, \geq 1 \mathrm{ng} / \mathrm{mL}$ ) and ovulation (Ovu) in response to the first GnRH injection (GnRH1) of the Ovsynch portion of the PresynchOvsynch protocols.

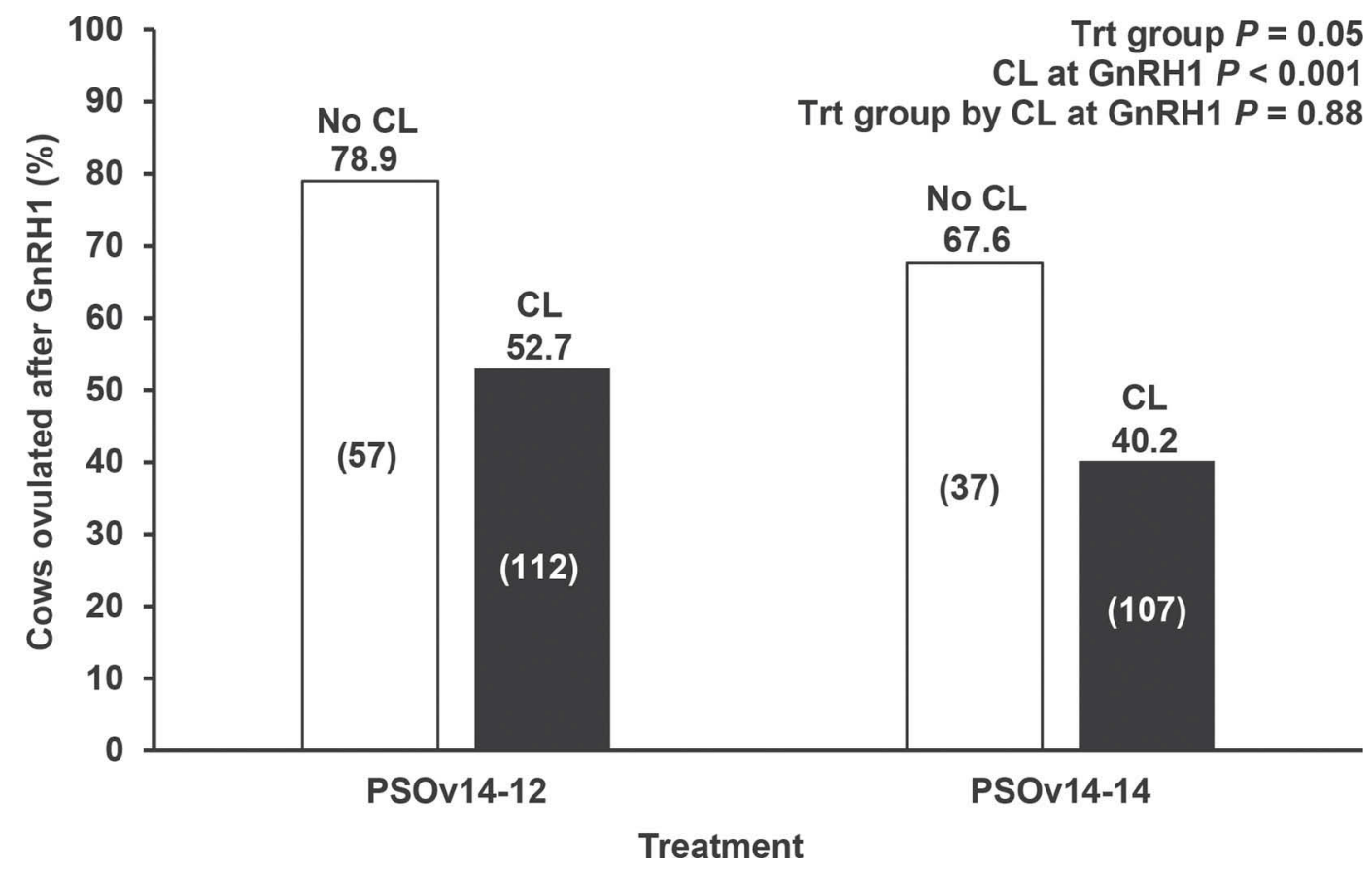

Figure 4. Proportion of cows ovulating in response to the first GnRH injection (GnRH1) of the Ovsynch portion of the Presynch-Ovsynch protocol for cows enrolled in Presynch-Ovsynch14-12 (PSOv14-12) and Presynch-Ovsynch14-14 (PSOv14-14) according to the presence of a functional corpus luteum (CL; progesterone $\geq 1 \mathrm{ng} / \mathrm{mL}$ ) at $\mathrm{GnRH} 1$. Trt $=$ treatment. 
to the presence of a CL not producing sufficient $\mathrm{P} 4$ to increase circulating $\mathrm{P} 4$ above $1 \mathrm{ng} / \mathrm{mL}$, or the presence of a recently regressed or regressing CL at the time of sampling. This is supported by the increased proportion of cows considered to have a functional CL when using a cutoff of $0.5 \mathrm{ng} / \mathrm{mL}$ of $\mathrm{P} 4$ to define a functional CL (85 and $82 \%$ for PSOv14-12 and PSOv14-14, respectively). Spontaneous luteolysis during Ovsynch was similar $(P$ $=0.71$ ) for cows in both treatments (17.1 and $17.9 \%$ for PSOv14-12 and PSOv14-14) but tended $(P=0.08)$ to be greater for multiparous than primiparous cows (21.2 vs. $12.4 \%$, respectively). The lack of difference between treatments coupled with a similar proportion of cows AIED during Ovsynch hinders the notion that the presence of a putative older CL at GnRH1 for cows in PSOv14-14 could be associated with greater rates of spontaneous luteolysis during Ovsynch. Conversely, the high rate of luteolysis for multiparous cows may in part explain the reduced fertility after TAI when compared with primiparous cows.

In spite of the greater ovulatory response to GnRH1 in PSOv14-12, no differences were observed in concentrations of P4 at PGF (Table 4), likely because of the greater proportion of cows with a functional CL at GnRH1 in PSOv14-14 and the similar rate of spontaneous luteolysis for both treatments during Ovsynch. The greater $(P=0.03)$ concentrations of $\mathrm{P} 4$ at $\mathrm{PGF}$ observed for primiparous compared with multiparous cows (3.3 vs. $2.8 \mathrm{ng} / \mathrm{mL}$, respectively) were expected because of the greater rate of luteolysis in multiparous cows that resulted in fewer $(6 \%$ less but not statistically significant) cows with a functional CL at PGF and the known effect of milk production on $\mathrm{P} 4$ metabolism (Sangsritavong et al., 2002). The same proportion of cows with a follicle $>10 \mathrm{~mm}(P=0.42)$ and a similar follicle size $(P=0.19)$ was observed for both treatments (Table 4). However, follicle size was greater $(P$ $<0.01)$ for multiparous than primiparous cows $(15.4$ vs. $14.0 \mathrm{~mm}$, respectively) and tended $(P=0.08)$ to be greater for cows that did not ovulate versus cows that ovulated to GnRH1 (15.3 vs. $14.3 \mathrm{~mm}$, respectively). Despite no statistical differences between parity groups for ovulation to GnRH1 (52.3 vs. $58.4 \%$ for multiparous and primiparous, respectively), a slightly greater proportion of primiparous cows ovulating to GnRH1 may explain the smaller follicle size for cows in this treatment because cows that ovulated to GnRH1 had smaller follicle size at PGF in this study.

\section{CONCLUSIONS}

Extending the interval from Presynch to Ovsynch from 12 to $14 \mathrm{~d}$ did not reduce $\mathrm{P} / \mathrm{AI}$ of lactating dairy cows that received TAI when cows detected in estrus any time after Presynch were inseminated. Ovulatory response to the first GnRH injection of the Ovsynch portion of Presynch-Ovsynch was reduced in the treatment with the extended interval from Presynch to Ovsynch. The presence of follicles not responsive to the LH surge generated by GnRH coupled with a reduction in $\mathrm{LH}$ release in cows with a CL at the time of the GnRH injection are the most likely reasons for the reduced ovulatory response. Nevertheless, the presence of a functional CL at GnRH1 seemed to benefit the fer-

Table 4. Ovarian parameters at the time of the prostaglandin $\mathrm{F}_{2 \alpha}$ (PGF) injection of the Ovsynch portion of the Presynch-Ovsynch protocol

\begin{tabular}{|c|c|c|c|}
\hline \multirow[b]{2}{*}{ Item } & \multicolumn{2}{|c|}{ Treatment $^{1}$} & \multirow[b]{2}{*}{$P$-value } \\
\hline & PSOv14-12 & PSOv14-14 & \\
\hline Cows with CL at PGF based on TUS $(\% ; \mathrm{n})$ & $\begin{array}{l}91.4 \\
(174)\end{array}$ & $\begin{array}{l}87.2 \\
(148)\end{array}$ & 0.22 \\
\hline Cows with $\mathrm{P} 4 \geq 1 \mathrm{ng} / \mathrm{mL}(\% ; \mathrm{n})$ & $\begin{array}{l}77.5 \\
(178)\end{array}$ & $\begin{array}{l}73.8 \\
(149)\end{array}$ & 0.42 \\
\hline Progesterone concentration $^{2}(\mathrm{ng} / \mathrm{mL} ; \mathrm{n})$ & $\begin{array}{c}3.1 \\
(178)\end{array}$ & $\begin{array}{c}3.0 \\
(149)\end{array}$ & 0.82 \\
\hline Cows with follicle $\geq 10 \mathrm{~mm}(\% ; \mathrm{n})$ & $\begin{array}{l}91.9 \\
(172)\end{array}$ & $\begin{array}{l}93.8 \\
(146)\end{array}$ & 0.42 \\
\hline Size of largest follicle ${ }^{3}(\mathrm{~mm} ; \mathrm{n})$ & $\begin{array}{l}14.5 \\
(172)\end{array}$ & $\begin{array}{l}15.2 \\
(146)\end{array}$ & 0.19 \\
\hline
\end{tabular}

${ }^{1}$ Lactating dairy cows received the Presynch-Ovsynch protocol (Presynch: PGF-14 d-PGF and Ovsynch: GnRH-7 d-PGF-56 h-GnRH- 16 to 20 h-TAI) with an interval of 12 (PSOv14-12) or 14 d (PSOv14-14) from the second PGF injection of Presynch to the first GnRH injection of Ovsynch. Cows detected in estrus any time between the second PGF injection of Presynch and the day of the TAI were inseminated. Transrectal ultrasonography of the ovaries was conducted and a blood sample collected at the time of the first GnRH and the PGF injection of the Ovsynch portion of the Presynch-Ovsynch protocol. CL = corpus luteum; TUS = transrectal ultrasonography; $\mathrm{P} 4=$ progesterone.

${ }^{2}$ Effect of parity group $(P=0.03)$.

${ }^{3}$ Effect of parity group $(P=0.01)$ and ovulation to the first GnRH of Ovsynch $(P=0.08)$. 
tility of cows receiving TAI more than ovulation rate, thereby compensating for the reduced fertility expected in cows failing to ovulate after GnRH. Dairy farms that combine insemination of cows after a detected estrus and TAI during the Presynch-Ovsynch protocol may benefit by using a 14-d interval between Presynch and Ovsynch by simplifying management without reducing reproductive performance of cows.

\section{ACKNOWLEDGMENTS}

The authors thank the commercial dairy farms that participated of this study for their collaboration and the use of their cows and facilities. We also thank Parnell Technologies Pty (Alexandria, Australia) for the support provided to conduct the study.

\section{REFERENCES}

Bruno, R. G., A. M. Farias, J. A. Hernandez-Rivera, A. E. Navarrette, D. E. Hawkins, and T. R. Bilby. 2013. Effect of gonadotropin-releasing hormone or prostaglandin F(2alpha)-based estrus synchronization programs for first or subsequent artificial insemination in lactating dairy cows. J. Dairy Sci. 96:1556-1567.

Caraviello, D. Z., K. A. Weigel, P. M. Fricke, M. C. Wiltbank, M. J. Florent, N. B. Cook, K. V. Nordlund, N. R. Zwald, and C. L. Rawson. 2006. Survey of management practices on reproductive performance of dairy cattle on large US commercial farms. J. Dairy Sci. 89:4723-4735.

Chebel, R. C., M. J. Al-Hassan, P. M. Fricke, J. E. Santos, J. R. Lima, C. A. Martel, J. S. Stevenson, R. Garcia, and R. L. Ax. 2010. Supplementation of progesterone via controlled internal drug release inserts during ovulation synchronization protocols in lactating dairy cows. J. Dairy Sci. 93:922-931.

Chebel, R. C., and J. E. Santos. 2010. Effect of inseminating cows in estrus following a presynchronization protocol on reproductive and lactation performances. J. Dairy Sci. 93:4632-4643.

Chebel, R. C., J. E. Santos, R. L. Cerri, H. M. Rutigliano, and R. G. Bruno. 2006. Reproduction in dairy cows following progesterone insert presynchronization and resynchronization protocols. J. Dairy Sci. 89:4205-4219.

Colazo, M. G., P. Ponce-Barajas, and D. J. Ambrose. 2013. Pregnancy per artificial insemination in lactating dairy cows subjected to 2 different intervals from presynchronization to initiation of Ovsynch protocol. J. Dairy Sci. 96:7640-7648.

Ferguson, J. D., and A. Skidmore. 2013. Reproductive performance in a select sample of dairy herds. J. Dairy Sci. 96:1269-1289.

Fricke, P. M., J. O. Giordano, A. Valenza, G. Lopes Jr., M. C. Amundson, and P. D. Carvalho. 2014. Reproductive performance of lactating dairy cows managed for first service using timed artificial insemination with or without detection of estrus using an activitymonitoring system. J. Dairy Sci. 97:2771-2781.

Galvão, K. N., M. F. Sa Filho, and J. E. Santos. 2007. Reducing the interval from presynchronization to initiation of timed artificial insemination improves fertility in dairy cows. J. Dairy Sci. 90:4212-4218.

Galvão, K. N., and J. E. Santos. 2010. Factors affecting synchronization and conception rate after the Ovsynch protocol in lactating Holstein cows. Reprod. Domest. Anim. 45:439-446.

Giordano, J. O., P. M. Fricke, J. N. Guenther, G. Lopes Jr., M. M. Herlihy, A. B. Nascimento, and M. C. Wiltbank. 2012a. Effect of progesterone on magnitude of the luteinizing hormone surge induced by two different doses of gonadotropin-releasing hormone in lactating dairy cows. J. Dairy Sci. 95:3781-3793.

Giordano, J. O., J. N. Guenther, G. Lopes Jr., and P. M. Fricke. 2012b. Changes in serum pregnancy-associated glycoprotein, pregnancy-specific protein $\mathrm{B}$, and progesterone concentrations before and after induction of pregnancy loss in lactating dairy cows. J. Dairy Sci. 95:683-697.

Giordano, J. O., M. C. Wiltbank, J. N. Guenther, R. Pawlisch, S. Bas, A. P. Cunha, and P. M. Fricke. 2012c. Increased fertility in lactating dairy cows resynchronized with Double-Ovsynch compared with Ovsynch initiated $32 \mathrm{~d}$ after timed artificial insemination. J. Dairy Sci. 95:639-653.

Lima, J. R., F. A. Rivera, C. D. Narciso, R. Oliveira, R. C. Chebel, and J. E. Santos. 2009. Effect of increasing amounts of supplemental progesterone in a timed artificial insemination protocol on fertility of lactating dairy cows. J. Dairy Sci. 92:5436-5446.

Moreira, F., C. Orlandi, C. A. Risco, R. Mattos, F. Lopes, and W. W. Thatcher. 2001. Effects of presynchronization and bovine somatotropin on pregnancy rates to a timed artificial insemination protocol in lactating dairy cows. J. Dairy Sci. 84:1646-1659.

NRC. 2001. Nutrient Requirements of Dairy Cattle. 7th ed. Natl. Acad. Sci., Washington, DC.

Sangsritavong, S., D. K. Combs, R. Sartori, L. E. Armentano, and M. C. Wiltbank. 2002. High feed intake increases liver blood flow and metabolism of progesterone and estradiol-17beta in dairy cattle. J. Dairy Sci. 85:2831-2842.

Sartori, R., J. M. Haughian, R. D. Shaver, G. J. M. Rosa, and M. C. Wiltbank. 2004. Comparison of ovarian function and circulating steroids in estrous cycles of Holstein heifers and lactating cows. J. Dairy Sci. 87:905-920.

Silva, E., R. A. Sterry, and P. M. Fricke. 2007. Assessment of a practical method for identifying anovular lactating dairy cows synchronized for first postpartum timed artificial insemination. J. Dairy Sci. 90:3255-3262.

Stevenson, J. S. 2011. Alternative programs to presynchronize estrous cycles in dairy cattle before a timed artificial insemination program. J. Dairy Sci. 94:205-217.

Stevenson, J. S., D. E. Tenhouse, R. L. Krisher, G. C. Lamb, J. E. Larson, C. R. Dahlen, J. R. Pursley, N. M. Bello, P. M. Fricke, M. C. Wiltbank, D. J. Brusveen, M. Burkhart, R. S. Youngquist, and H. A. Garverick. 2008. Detection of anovulation by heatmount detectors and transrectal ultrasonography before treatment with progesterone in a timed insemination protocol. J. Dairy Sci. 91:2901-2915. 